The findings of Tanaka and colleagues ${ }^{4}$ should stimulate further efforts in the study of nematic LCs confined to porous media. For instance, it would be interesting to classify and quantify the number of metastable states as a function of the geometry of the confining medium. Also, going beyond the Lebwohl-Lasher description of the nematic LC, and substituting Monte Carlo quasi-time dynamics with a more realistic approach should provide better insight into dynamical processes. What is more exciting, the fabrication of devices that can take advantage of the memorization capabilities poses a fascinating challenge: will it be possible to optimally manipulate the memory of such a device to read and write (individual) bits of information by external fields?

Igor Muševič and Slobodan Žumer are at the Faculty of Mathematics and Physics, University of Ljubljana, Jadranska 19, Ljubljana, Slovenia, and the J. Stefan Institute, Jamova 39,

Ljubljana, Slovenia.

e-mail: igor.musevic@ijs.si; slobodan.zumer@fmf.uni-lj.si
References

1. Poulin, P., Stark, H., Lubensky, T. C. \& Weitz, D. A. Science 275, 1770-1773 (1997).

2. Muševič, I., Škarabot, M., Tkalec, U., Ravnik, M. \& Žumer, S Science 313, 954-958 (2006)

3. Smalyukh, I. I., Lansac, Y., Clark, N. A. \& Trivedi, R. P. Nature Mater. 9, 139-145 (2010).

4. Araki, T., Buscaglia, M., Bellini, T. \& Tanaka, H. Nature Mater. 10, 303-309 (2011)

5. Kuksenok, O. V., Ruhwandl, R. W., Shiyanovskii, S. V. \& Terentjev, E. M. Phys. Rev. E 54, 5198-5203 (1996).

6. Stark, H. Phys. Rep. 351, 387-474 (2001).

7. Ravnik, M. et al. Phys. Rev. Lett. 99, 247801 (2007).

8. Kang, D., Maclennan, J. E., Clark, N. A., Zakhidov, A. A. \& Baughman, R. H. Phys. Rev. Lett. 86, 4052-4055 (2001)

9. Pasini, P. \& Zannoni, C. (eds) Advances in the Computer Simulation of Liquid Crystals (Kluwer, 2000).

10. Diep, H. T. (ed.) Frustrated Spin Systems (World Scientific, 2004).

\title{
SOUND SENSE OF DIRECTION
}

Wave sculpting has become one of the unanticipated unifying goals of modern physical science. Comparable principles, involving the breaking of symmetry in time or space, apply to the tailoring of wavefunctions in molecular dynamics, the shaping of light waves by photonic crystals, the creation of 'invisibility shields' over a wide range of electromagnetic wavelengths, the focusing required for X-ray microscopes, plasmon engineering for subwavelength optics, and even speculative schemes for harnessing wave power or protecting structures from seismic waves.

In many of these examples, the trick is to control wave-scattering phenomena using regular arrays of scattering objects, commonly called metamaterials. The manipulation of acoustic waves in this way is not only one of the most accessible wavesculpting technologies - because acoustic wavelengths demand macroscopic 'sonic crystals' that are relatively easy to make and control but is also of clear practical value, whether employed for reducing environmental noise or for enhancing the performance and capabilities of ultrasound techniques in biomedical imaging. Furthermore, as a bonus, sonic crystals can be rather beautiful ${ }^{1}$.

The latest manifestation of the art, reported by Li et al. ${ }^{2}$, is a sonic crystal that acts as a diode, producing unidirectional transmission of sound. This is not the first description of such a device, but the new design is potentially cheaper, more versatile and more efficient. Previously, Liang et al. made an 'acoustic rectifier' that combined a sonic crystal (alternating layers of water and glass) with a nonlinear medium (a microbubble suspension $)^{3,4}$. Li et al. have now dispensed with the need for acoustic nonlinearity by using a carefully shaped array of scatterers here steel square-prism columns. Both groups happen to be based at Nanjing University in China.

The sonic crystal achieves unidirectional flow by means of sawtooth spatial asymmetry in the arrangement of columns. The result is that sound within particular frequency bands - here of tens of kilohertz, reaching from the upper limits of human hearing into the ultrasonic - is (mostly) propagated when incident from one direction, but more or less wholly reflected when coming from the opposite direction. The sound waves exiting the device are not parallel to those entering, showing that they are in fact reconstructed by interference of other propagating modes, which is how they effectively evade the acoustic bandgap.

Among the advantages of the new design, apart from the ease of manufacture and handling, are that the low conversion efficiencies (that is, low transmission) typical of nonlinear acoustic media

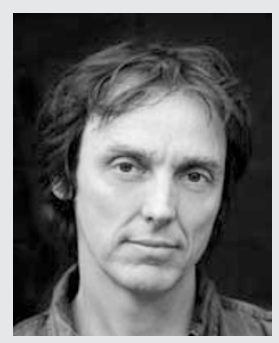

PHILIP BALL

are avoided and that the 'diode' permits broadband unidirectional flow. Another limitation of the nonlinear device ${ }^{4}$ is that it alters the frequency of the transmitted sound. Furthermore, the acoustic flow in the diode of Li et al. can be conveniently manipulated simply by rotating the square pillars: a twist of $45^{\circ}$ can switch the transmission within one wavelength band between 'on' and 'off'. This sort of structural change could presumably be easily mechanized to open and close an acoustic diode at will, or to alter or modulate the blocked bandwidth. The researchers hope that such devices might eventually be deployed in acoustic circuits that permit the direct logical processing of sound.

References

1. Martínez-Sala, R. et al. Nature 378, 241 (1995).

2. Li, X-F. et al. Phys. Rev. Lett. 106, 084301 (2011)

3. Liang, B., Yuan, B. \& Cheng, J-C. Phys. Rev. Lett. 103, 104301 (2009).

4. Liang, B., Guo, X. S., Tu, J., Zhang, D. \& Cheng, J. C Nature Mater. 9, 989-992 (2010). 